\title{
Combined Effects of Thermal Radiation and Hall Current on MHD Free-Convective Flow and Mass Transfer over a Stretching Sheet with Variable Viscosity
}

\author{
G.C. Shit ${ }^{\dagger}$ and R. Haldar \\ Department of Mathematics, Jadavpur University, Kolkata-700032, India \\ †Corresponding Author Email: gcs@math.jdvu.ac.in
}

(Received March 8, 2009; accepted March 28, 2009)

\begin{abstract}
An analysis has been carried out on the effects of thermal radiation and Hall current of a magneto hydrodynamic freeconvective flow and mass transfer over a stretching sheet with variable viscosity in the presence of heat generation/absorption. The fluid viscosity is assumed to vary as an inverse linear function of temperature. The boundary-layer equations governing the flow problem under consideration have been reduced to a system of nonlinear ordinary differential equations by employing a similarity transformation. Using the finite difference scheme, numerical solutions to the transform ordinary differential equations have been obtained and the results are presented graphically. The numerical results obtained are in good agreement with the existing scientific literature.
\end{abstract}

Keywords: Thermal radiation; Variable viscosity; MHD; Hall current; Heat and Mass transfer.

\section{NOMENCLATURE}

$\begin{array}{ll}a, T_{r} & \text { are constant } \\ \vec{B} & \text { magnetic induction vector } \\ B_{0} & \text { strength of applied magnetic field } \\ C & \text { dimensional concentration variable } \\ C_{f} & \text { local skin-friction coefficient } \\ c_{p} & \text { specific heat at constant pressure } \\ D & \text { thermal molecular diffusivity } \\ \vec{E} & \text { intensity vector of the electric field } \\ e & \text { charge of the electron } \\ G_{c} & \text { modified Grashof number } \\ G r & \text { Grashof number } \\ g_{0} & \text { acceleration due to gravity } \\ \vec{J} & \text { electric current density vector } \\ K & \text { mean absorption coefficient } \\ k & \text { thermal conductivity } \\ k_{0} & \text { measures the rate of reaction } \\ M & \text { magnetic parameter } \\ m & \text { Hall current parameter } \\ N r & \text { thermal radiation parameter } \\ N u & \text { local Nusselt number } \\ n & \text { order of chemical reaction } \\ n_{e} & \text { number density of the electron }\end{array}$

\section{INTRODUCTION}

In the recent past there has been a growing interest in boundary-layer flow on a continuous moving surface in the presence of magnetic field with or without considering the effect of Hall current. These are very significant types of flow encountered in several

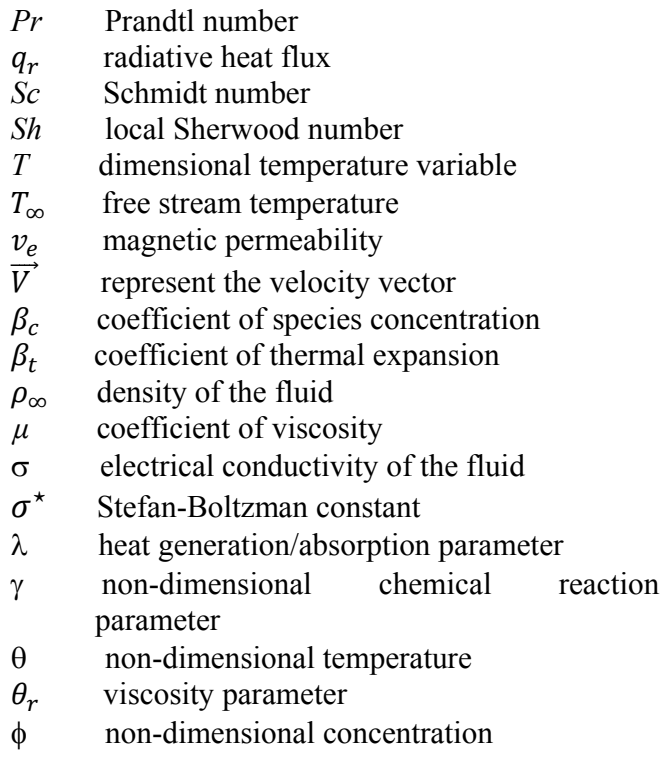

engineering applications, such as in polymer processing, gas turbines, various propulsion device for aircraft, space vehicles, electro-chemistry, MHD power generators, flight magneto hydrodynamics as well as in the field of planetary magnetosphere, aeronautics and chemical engineering. If the temperature of the 
surrounding fluid becomes high, then the thermal radiation effect play a vital role in the case of space technology.

Sakiadis (1961) first explored the study of boundary layer flow on a continuous moving surface and Crane (1970) extended this problem to a stretching sheet whose surface velocity varies linearly with the distance $x$ from a fixed origin. During the past decades several investigators have considered the boundary-layer flow problems under different physical situations. Gupta and Gupta (1977) examined the heat and mass transfer over a stretching sheet subject to suction or blowing. The influence of a uniform magnetic field on the flow of an electrically conducting fluid past a stretching sheet have been investigated by Pavlov (1974), Andersson (1992), Andersson et al.(1992), Chakrabarty and Gupta (1979), Char (1994), Watanabe and Pop (1993). Afify (2004) investigated the chemical reaction on free-convective flow and mass transfer of a viscous, incompressible and electrically conducting fluid over a stretching sheet in the presence of a uniform transverse magnetic field. In all these investigations, the electrical conductivity of the fluid was assumed to be uniform and the magnetic field intensity was assumed to be low. However, in an ionized fluid where the density is low and thereby magnetic field intensity is very strong, the conductivity normal to the magnetic field is reduced due to the spiraling of electrons and ions about the magnetic lines of force before collisions take place and a current induced in a direction normal to both the electric and magnetic fields. This phenomenon is known as Hall Effect. Watanabe and Pop (1995) investigated the magneto hydrodynamic boundary layer flow over a continuously moving semi-infinite flat plate by taking into account the Hall currents. Shit (2009) studied the effects of Hall current on MHD free-convective flow and mass transfer over a stretching sheet in the presence of chemical reaction.

Recently, a new idea is added to the study of boundarylayer fluid flow and heat transfer. Indeed, in such studies, the effects of thermal radiation and temperature dependent viscosity are also included. Many processes in engineering applications occur at high temperatures and the radiate heat transfer becomes very important for the design of the pertinent equipment. In view of this, Rapits and Perdikis (1998) studied the flow of a viscoelastic fluid past a stretching sheet in the presence of thermal radiation. Mukhopadhaya et al. (2005) investigated the problem of MHD boundary-layer flow over a heated stretching sheet with variable viscosity. The radiation effect on boundary-layer fluid flow with or without applying magnetic field under different situations were studied by Shateyi (2008), Mahmoud (2009) and Sharma and Singh (2009). Salem (2007) investigated the effect of variable viscosity on MHD viscoelastic fluid flow and heat transfer over a stretching sheet without considering thermal radiation effect. But, no attempt is available in the existing scientific literatures for the consideration of the combined effect of thermal radiation and Hall current on the study of MHD boundary-layer flow. Thus, the present study fills the gap in these directions. Again, combined heat and mass transfer problems with chemical reaction are of increasing importance in many processes, like drying, evaporation at the surface of a water body, energy transfer in a wet cooling tower etc. In this context, Muthucumarswamy and Ganesan (2001) studied the effect of the chemical reaction and injection as well as flow characteristics in an unsteady upward motion of an isothermal plate. Chamakha (2003) carried out the MHD flow of uniformly stretching vertical permeable surface in the presence of heat generation/absorption along with the chemical reaction. Very recently, Mohamed and Abo-Dahab (2009) have investigated the influence of chemical reaction and thermal radiation on hydro magnetic free convective heat and mass transfer for a micro polar fluid. Wherein he considered a porous medium bounded by an infinite vertical porous plate in the presence of heat generation. In all these studies ignores the effect of the consideration of Hall current.

Now we propose to study the combined effects of thermal radiation and Hall current on the hydro magnetic free-convective flow and mass transfer over a stretching surface with variable viscosity in the presence of heat generation/absorption. The present problem pertains to situation in which the n-th order chemical reaction takes place. Since the governing differential equations are highly nonlinear, Newton's linearization method is used followed by a finite difference scheme to obtain the numerical solutions. The effects of various parameters on the velocity, temperature and concentration as well as on the local skin-friction coefficient, local Nusselt number and local Sherwood number are presented.

\section{MATHEMATICAL ANALysis}

We consider the steady free-convective flow and mass transfer of an incompressible, viscous and electrically conducting fluid past a stretching sheet and the sheet is stretched with a velocity proportional to the distance from a fixed origin $\mathrm{O}$ (cf. Fig. 1).

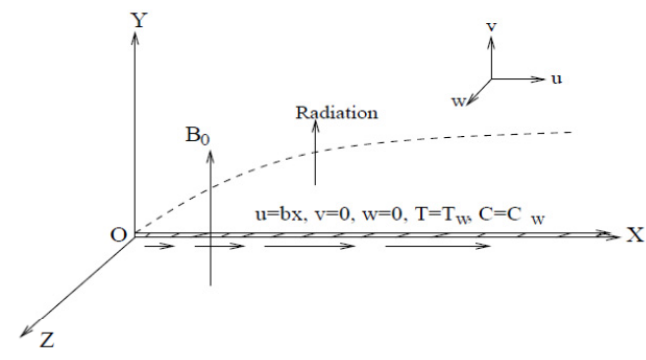

Fig.1 Physical sketch of the problem

A uniform strong magnetic field of strength $B 0$ is imposed along the $y$-axis and the effect of Hall current is taken into account. Taking Hall effects into account the generalized Ohm's law (Cowling 1957) may be put in the following form

$\vec{J}=\frac{\sigma}{1+m^{2}}\left(\vec{E}+\vec{V} * \vec{B}-\frac{1}{e n_{e}} \vec{J} * \vec{B}\right)$

Where $m=\frac{\sigma B 0}{e n_{c}}$ is defined as the Hall current parameter. A very interesting fact that the effect of Hall current gives rise to a force in the $z$-direction which in turn produces a cross-flow velocity in this direction and 
then the flow becomes three-dimensional. The temperature and the species concentration are maintained at a prescribed constant values $T_{w}, C_{w}$ at the sheet and $T_{\infty}$ and $C_{\infty}$ are the fixed values far away from the sheet. Since the concentration of diffusing species is very small in comparison to other chemical species, the Soret and Dufour effects have been neglected.

Following Lai and Kulacki (1990), the fluid viscosity $\mu$ is assumed to vary as a reciprocal of a linear function of temperature given by

$\frac{1}{\mu}=\frac{1}{\mu_{\infty}}\left[1+\gamma_{0}\left(T-T_{\infty}\right)\right]$

or

$\frac{1}{\mu_{\infty}}=a\left(T-T_{r}\right)$

Where $a=\frac{\gamma_{0}}{\mu_{\infty}}$ and $T_{r}=T_{\infty}-\frac{1}{\gamma_{0}}$.

In the above equation both $a$ and $T_{r}$ are constants, and their values depend on the thermal property of the fluid, i.e., $g_{0}$. In general $a>0$ represent for liquids, whereas for gases $a<0$.

By assuming Rosseland approximation for radiation, the radiative heat flux $q_{r}$ is given by

$q_{r}=-\frac{4 \sigma^{\star}}{3 K} \frac{\partial T^{4}}{\partial y}$

Where $\sigma^{\star}$ is the Stefan-Boltzman constant and $K$ is the mean absorption coefficient. We assume that the temperature differences within the flow are sufficiently small such that $T^{4}$ may be expressed as a linear function of the temperature as shown in Chamakha (2003). Expanding $T^{4}$ in a Taylor series about $T_{\infty}$ and neglecting higher order terms, we obtain

$T^{4} \simeq 4 T_{\infty}^{3} T-3 T_{\infty}^{4}$

Substituting $T^{4}$ from Eq. (4) in Eq. (3) and differentiating the resulting equation with respect to $y$, we obtain as

$\frac{\partial q_{r}}{\partial y}=-\frac{16 \sigma^{\star} T_{\infty}^{3}}{3 K} \frac{\partial^{2} T}{\partial y^{2}}$

Owing to the above mentioned assumptions, the boundary layer free-convection flow with mass transfer and generalized Ohm's law with Hall current effect are governed by the following system of equations:

$\frac{\partial u}{\partial x}+\frac{\partial v}{\partial y}=0$

$\rho_{m}\left(u \frac{\partial u}{\partial x}+v \frac{\partial u}{\partial y}\right)=\frac{\partial}{\partial y}\left(\mu \frac{\partial u}{\partial y}\right)+\rho_{m} g_{0} \beta_{r}(T-$

$\left.T_{\infty}\right)+\rho_{m} g_{0} \beta_{c}\left(C-C_{\infty}\right)-\frac{\sigma B_{0}^{2}}{1+m^{2}}(u+m w)$

$\rho_{m}\left(u \frac{\partial w}{\partial x}+v \frac{\partial w}{\partial y}\right)=\frac{\partial}{\partial y}\left(\mu \frac{\partial w}{\partial y}\right)+\frac{\sigma B_{0}^{2}}{1+m^{2}}(m u-w)$

$\rho_{m} C_{p}\left(u \frac{\partial T}{\partial x}+v \frac{\partial T}{\partial y}\right)=k \frac{\partial^{2} T}{\partial y^{2}}-Q\left(T-T_{\infty}\right)-\frac{\partial q_{r}}{\partial y}$

$u \frac{\partial C}{\partial x}+v \frac{\partial C}{\partial y}=D \frac{\partial^{2} C}{\partial y^{2}}-k_{0}\left(C-C_{\infty}\right)^{n}$

Where $(\mathrm{u}, \mathrm{v}, \mathrm{w})$ are the velocity components along the $(x ; y ; z)$ directions respectively.
The boundary conditions for the present problem can be written as

$u=b x, v=w=0, \mathrm{~T}=\mathrm{T}_{\mathrm{w}}, \mathrm{C}=\mathrm{C}_{\mathrm{w}}$ at $\mathrm{y}=0$
$u \rightarrow 0, w \rightarrow 0, T \rightarrow T_{\infty}, C \rightarrow C_{\infty}$ as $y \rightarrow \infty$

Where $b(>0)$ being stretching rate of the sheet. The boundary conditions on velocity in Eq. (11) are the noslip condition at the surface $y=0$, while the boundary conditions on velocity at $y \rightarrow \infty$ follow from the fact that there is no flow far away from the stretching surface.

To examine the flow regime adjacent to the sheet, the following transformations are invoked

$u=b x f^{\prime}(\eta) ; v=-\sqrt{b v} f(\eta) ; w=b x g(\eta) ;$

$\eta=\sqrt{\frac{b}{v}} y ; \theta(\eta)=\frac{T-T_{\infty}}{T_{w}-T_{\infty}} ; \phi=\frac{C-C_{\infty}}{C_{w}-C_{\infty}}$

Where $f$ is a dimensionless stream function, $\mathrm{h}$ is is the similarity space variable, $\theta$ and $\phi$ are the dimensionless temperature and concentration respectively. Clearly, the continuity Eq. (6) is satisfied by $u$ and $v$ defined in Eq. (13). Substituting Eq. (13) in Eq. (7) to Eq. (10) gives

$$
\begin{aligned}
& \left(\frac{\theta-\theta_{r}}{\theta_{r}}\right)\left(f^{\prime 2}-f f^{\prime \prime}\right)+f^{\prime \prime \prime}-\left(\frac{\theta^{\prime}}{\theta-\theta_{r}}\right) f^{\prime \prime}- \\
& \left(\frac{\theta-\theta_{r}}{\theta_{r}}\right)(G r \theta+G c \phi)+M\left(\frac{\theta-\theta_{r}}{\theta_{r}}\right)\left(\frac{f^{\prime}+m g}{1+m^{2}}\right)=0 \\
& \left(\frac{\theta-\theta_{r}}{\theta_{r}}\right)\left(f^{\prime} g-f g^{\prime}\right)+g^{\prime \prime}-\left(\frac{\theta^{\prime}}{\theta-\theta_{r}}\right) g^{\prime}- \\
& M\left(\frac{\theta-\theta_{r}}{\theta_{r}}\right)\left(\frac{m f^{\prime}-g}{1+m^{2}}\right)=0 \\
& (3 N r+4) \theta^{\prime \prime}+3 N r \operatorname{Pr} \lambda \theta=0 \\
& \phi^{\prime \prime}+S c\left(f \phi^{\prime}-\gamma \phi "\right)=0
\end{aligned}
$$

Similarly, the transformed boundary conditions are given by

$$
\begin{array}{ll}
f^{\prime}(\eta)=1, \quad f(\eta)=0, \quad g(\eta)=0, & \theta(\eta)= \\
1, \phi(\eta)=1 \quad \text { at } \eta=0 & \\
f^{\prime}(\eta) \rightarrow 0, \quad g(\eta) \rightarrow 0, \quad \theta(\eta) \rightarrow 0, & \phi(\eta) \rightarrow \\
0 \quad \text { as } \quad \eta \rightarrow \infty &
\end{array}
$$

Where prime denotes the differentiation with respect to $\mathrm{h}$ only and the dimensionless parameters appearing in the Eq. (14) - Eq. (17) are respectively defined as $\theta_{r}=\frac{T_{r}-T_{\infty}}{T_{w}-T_{\infty}}=-\left[\frac{1}{\gamma_{0}\left(T_{w}-T_{\infty}\right)}\right]$ the viscosity parameter, $M=\frac{\sigma B_{0}^{2}}{\rho_{\infty} b}$ the magnetic parameter, $\operatorname{Pr}=\frac{\rho_{\infty} C_{p} v}{k}$ the Prandtl number, $\gamma=\frac{k_{0}}{b}\left(C_{w}-c_{\infty}\right)^{n-1}$ the nondimensional chemical reaction parameter, $G_{r}=$ $\frac{g_{0} \beta_{r}\left(T_{w}-T_{\infty}\right)}{b^{2} x}$ the local Grashof number, $G_{c}=$ $\frac{g_{0} \beta_{c}\left(C_{w}-C_{\infty}\right)}{b^{2} x}$ the local modified Grashof number, $N r=\frac{k K}{4 T_{\infty}^{3} \sigma^{*}}$ the thermal radiation parameter, $S c=\frac{\mu}{\rho_{\infty} D}$ the Schmidt number and $\lambda=\frac{Q}{\rho_{\infty} C_{p} b}$ is defined as the heat generation/absorption parameter.

The important characteristics of the present investigation are the local skin-friction coefficient $C_{f}$, the local Nusselt number $N u$ and the local Sherwood number $S h$ defined by 
$C_{f}=\frac{\tau_{w}}{\mu b x \sqrt{\frac{b}{v}}}=f^{\prime \prime}(0)$

where

$\tau_{w}=\mu(\partial u / \partial y)_{y=0}=\mu b x \sqrt{\frac{b}{v}} f^{\prime \prime}(0)$,

$N u=\frac{q_{w}}{k \sqrt[b]{\frac{b}{v}}\left(T_{w}-T_{\infty}\right)}$

where

$q_{w}=-k(\partial T / \partial y)_{y=0}=-k \sqrt{\frac{b}{v}}\left(T_{w}-T_{\infty}\right) \theta^{\prime}(0)$

and

$S h=\frac{m_{w}}{D \sqrt{\frac{b}{v}}\left(C_{w}-C_{\infty}\right)}=-\phi^{\prime}(0)$

where

$m_{w}=-D(\partial C / \partial y)_{y=0}=-D \sqrt{\frac{b}{v}}\left(C_{w}-C_{\infty}\right) \phi^{\prime}(0)$

If we consider $M=m=0, \theta_{r} \rightarrow \infty$ and $\mathrm{Nr}=\mathrm{Sc}=G_{r}=$ $G_{c}=0$, the present flow problem becomes hydrodynamic boundary-layer flow past a stretching sheet whose analytical solution put forwarded by Crane (1970) as follows

$f(\eta)=1-e^{-\eta} \quad$ i.e,$\quad f^{\prime}(\eta)=e^{-\eta}$

An attempt has been made to validate our results for the axial velocity $f^{\prime}(\eta)$. We compared our results with this analytical solution and found excellent agreement.

\section{RESUlt AND Discussion}

The system of coupled and non-linear ordinary differential Eq. (14) to Eq. (17) along with the boundary conditions (18) and (19) have been solved numerically by employing a finite difference scheme. We used Newton's linearization method (cf. Cebeci and Cousteix (1999)) to linearize the discretized equations. The essential features of this technique is that it is based on a finite difference scheme, which has better stability, simple, accurate and more efficient. Finite difference technique leads to a system which is tri-diagonal and therefore speedy convergence as well as economical memory space to store the coefficients. The computational work has been carried out by taking $\delta \eta=$ 0:0125 (the step size along $\eta$ direction) and further reduction in $\delta \eta$ does not bring about any significant change. In the present study, the numerical values to the physical parameters have been chosen so that $M ; m$; $N r ; \theta_{r} ; \operatorname{Pr} ; G r ; G c ; S c ; n ; \gamma$ and $\lambda$ are varied over a range, which are listed in the figure legends (cf. Watanabe and Pop (1993, 1995), Afify 2004 and Salem 2007). Figure 2 shows that our numerical results are complete agreement with those of Crane (1970).

Figures 3 - 8 illustrate the variation of axial velocity for different values of the dimensionless parameters that involved in the present study. Fig. 3 shows that the axial velocity decreases with the increase of the magnetic parameter $M$, whereas from Fig. 4, it indicates that the axial velocity increases with the increase of Hall parameter $m$.

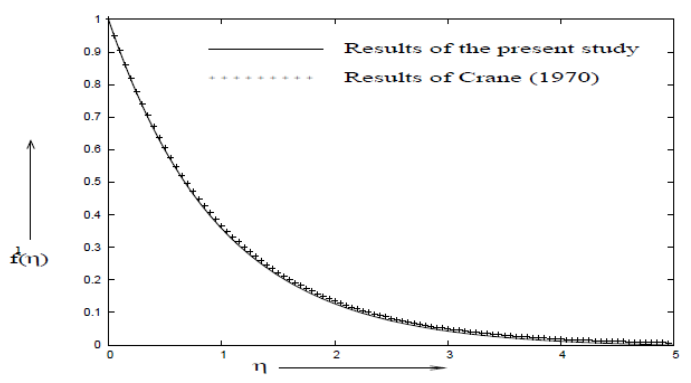

Fig. 2. Comparison of axial velocity profile $f^{\prime}(\eta)$ with the results of Crane (1970) $\mathrm{M}=\mathrm{Gr}=\mathrm{Gc}=0.0$ $\theta \rightarrow \infty, \mathrm{Sc}=\mathrm{Pr}=\mathrm{Nr}=0.0$

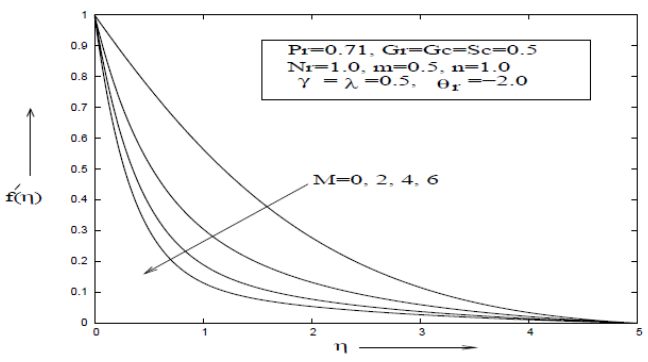

Fig. 3. Variation of $f^{\prime}(\eta)$ with $\eta$ for different values of $\mathrm{M}$

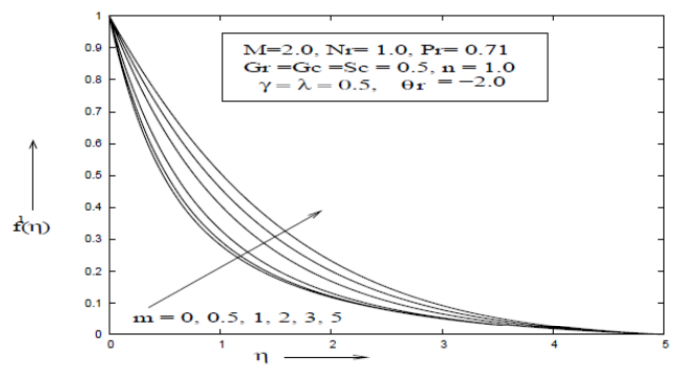

Fig. 4. Variation of $f^{\prime}(\eta)$ with $\eta$ for different values of $\mathrm{m}$

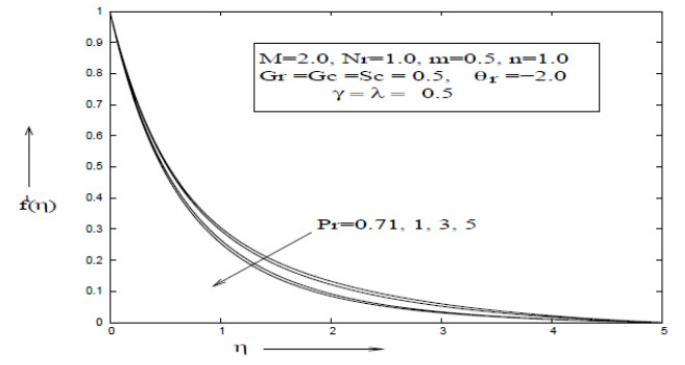

Fig. 5. Variation of $f^{\prime}(\eta)$ with $\eta$ for different values of $\operatorname{Pr}$

This is due to the fact that as $M$ increases, the Lorentz force which opposes the flow and leads to the deceleration of fluid motion. The anomalous behavior in $f^{\prime}(\eta)$ with the variation of $m$ is observed due to the presence of Hall current and thereby induces a crossflow velocity component $g(\eta)$. It has been shown in Fig. 5 and Fig. 6 that the axial velocity decreases with the increase of the Prandtl number $P r$ as well as the 


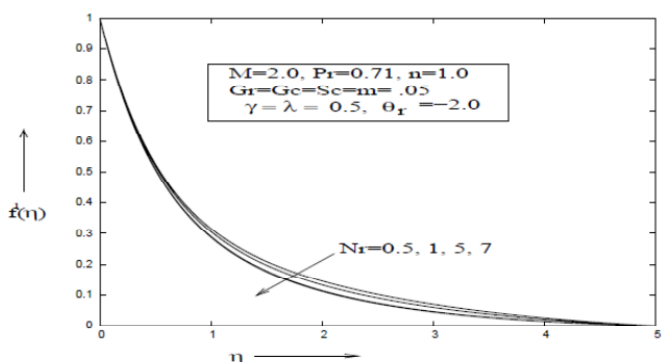

Fig. 6. Variation of $f^{\prime}(\eta)$ with $\eta$ for different values of $\mathrm{Nr}$

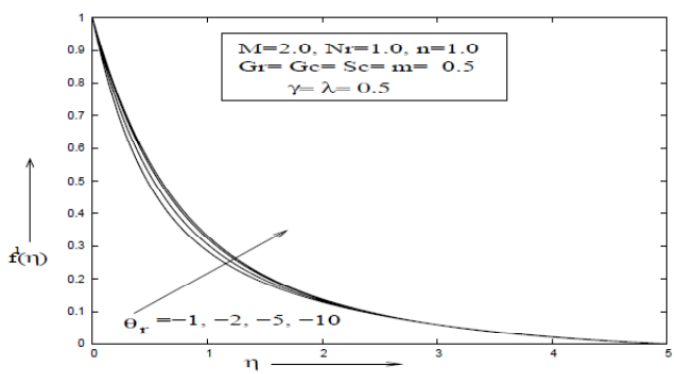

Fig. 7. Variation of $f^{\prime}(\eta)$ with $\eta$ for different values of $\theta_{r}$

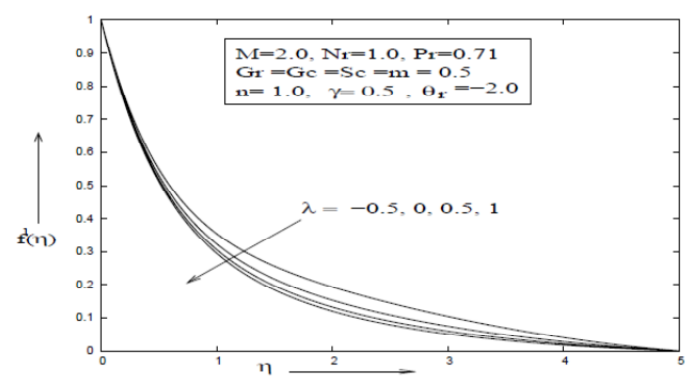

Fig. 8. Variation of $f^{\prime}(\eta)$ with $\eta$ for different values of $\lambda$

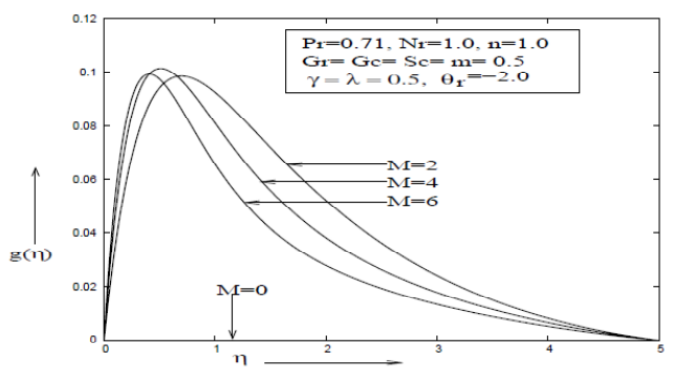

Fig. 9. variation of $\mathrm{g}(\eta)$ with $\eta$ for different values of $\mathrm{M}$

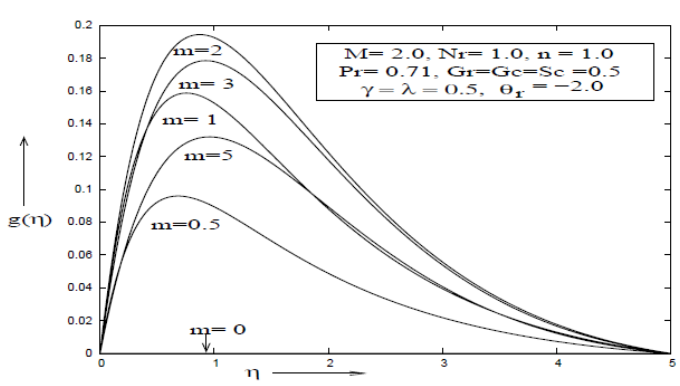

Fig. 10. Variation of $\mathrm{g}(\eta)$ for different values of the Hall parameter $\mathrm{m}$

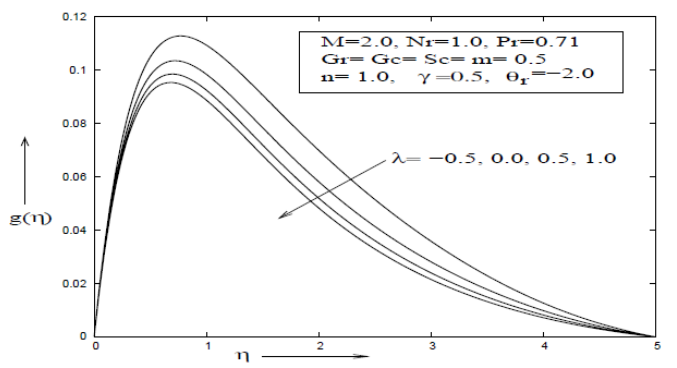

Fig. 11. Variation of $\mathrm{g}(\eta)$ with $\eta$ for different values of $\lambda$

thermal radiation parameter $N r$. This shows that there would be a decrease of boundary-layer thickness in the presence of thermal radiation. Fig. 7 depicts that the axial velocity $f^{\prime}(\eta)$ increases with the decreasing of the viscosity parameter $\theta r$. This observation leads to an increase of the thermal boundary-layer thickness. The effects of heat generation parameter $(\lambda>0)$ and absorption parameter $(\lambda<0)$ on the axial velocity presented in Fig. 8. This figure shows that the axial velocity decreases as the parameter $\lambda$ increases.

Figures 9-11 give the distribution of the $z$ - component of velocity, which is induced due to the presence of Hall effects. All these figures show that for any particular values of the physical parameters $g(\eta)$ reaches a maximum value at a certain height $\eta$ above the sheet and beyond which $g(\eta)$ decreases gradually in asymptotic nature. It is noticed from Fig. 9 that in the absence of magnetic field $(M=0)$ cross flow velocity vanishes. This is due to fact that, when there is no applied magnetic field, the cross flow velocity would not arise. The variation of Hall current parameter $m$ on the cross-flow velocity $g(\eta)$ shown in Fig. 10. An interesting result observed from this figure that the cross-flow velocity gradually increases with the increase of $m>2$ and the velocity decreases for $m>2$. The values of $m$ beyond which the flow behavior changes are considerably depend upon the choice of the magnetic parameter $M$. Thus we conclude that after certain magnetic field strength the flow behavior is significantly affected. We observed from Fig. 11 that the cross-flow velocity decreases with the increase of the heat generation/absorption parameter $\lambda$. Thus the parameter $\lambda$ gives rise to the decrease of the momentum boundary-layer thickness.

The distribution of dimensionless temperature $\theta(\eta)$ along the height of the stretching sheet for different values of the dimensionless parameters involved in the present study displayed through Figs. 12-16. Fig. 12 shows that the temperature of the fluid enhanced by the application of an external magnetic field. While the effect of the Hall current parameter $m$ has a reducing effect on the dimensionless temperature $\theta(\eta)$ shown in Fig. 13. It may note that the effect of Hall current parameter $m$ opposes the effect of magnetic field on the temperature distribution. Fig. 14 presents the variation of Prandtl number $P r$ on the temperature $\theta(\eta)$. The results presented in Fig. 14 shows that the dimensionless temperature decreases as the Prandatl number $\operatorname{Pr}$ increases. This is due to the fact that for 
smaller values of $P r$ are equivalent to larger values of thermal conductivities and therefore heat is able to diffuse away from the stretching sheet.

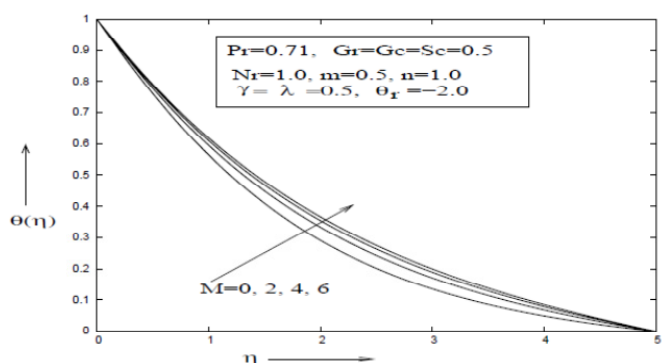

Fig. 12. Distribution of dimensionless temperature $(\eta)$ with $\eta$ for different values of $\mathrm{M}$

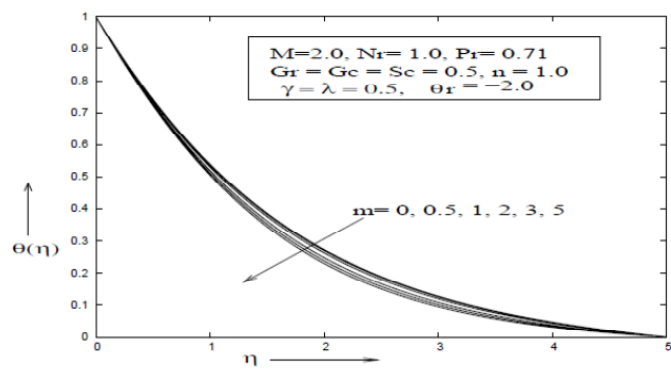

Fig.13. Distribution of dimensionless temperature $(\eta)$ with $\eta$ for different values of $m$

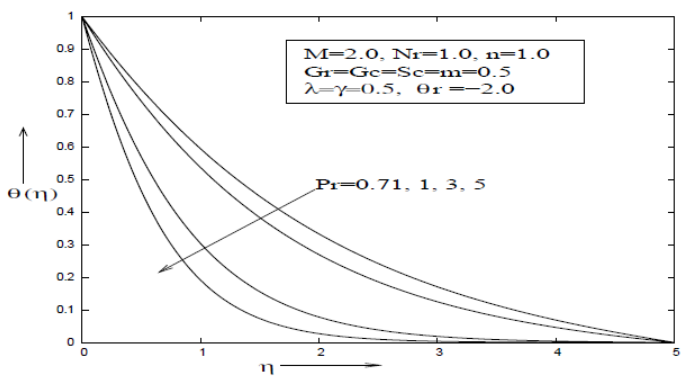

Fig. 14. Distribution of dimensionless temperature $(\eta)$ with $\eta$ for different values of $\operatorname{Pr}$

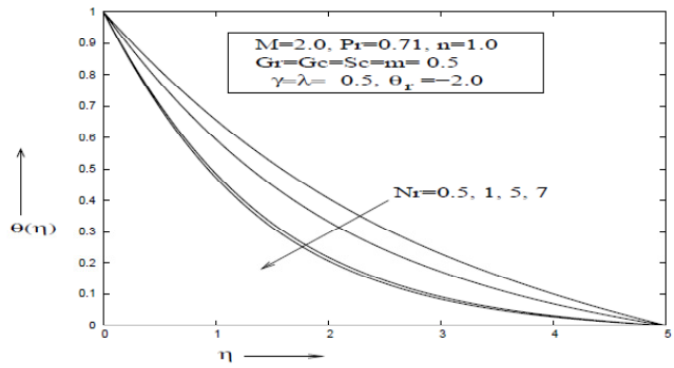

Fig. 15. Distribution of dimensionless temperature $(\eta)$ with $\eta$ for different values of $\mathrm{Nr}$

Fig. 15 represents the temperature profiles for various values of the thermal radiation parameter $\mathrm{Nr}$ in the boundary-layer. Increasing the thermal radiation parameter $\mathrm{Nr}$ produces a decrease in the temperature of the fluid. This is because of the fact that the thermal boundary-layer thickness decreases with increasing the thermal radiation parameter $N r$. The effect of heat generation/absorption parameter $\lambda$ on the dimensionless temperature $\theta(\eta)$ is shown in Fig. 16. It is clear that an increase in the heat generation/absorption parameter $\lambda$ leads to a decrease of $\theta(\eta)$. Thus the effect of internal heat generation is to decrease the rate of energy transport to the fluid, thereby decreasing the temperature of the fluid.

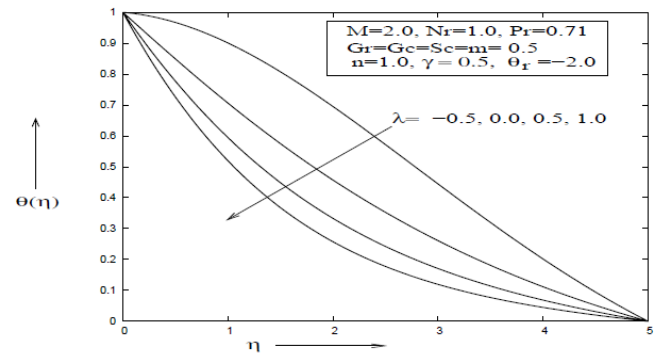

Fig. 16. Distribution of dimensionless temperature $(\eta)$ with $\eta$ for different values of $\lambda$

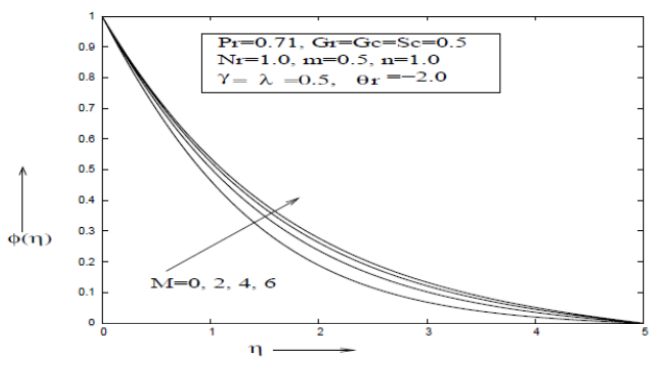

Fig. 17. Influence of concentration species $(\eta)$ with $\eta$ for different values of $\mathrm{M}$

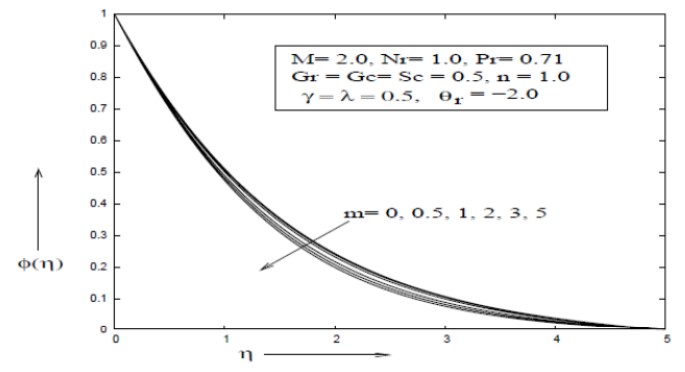

Fig. 18. Influence of concentration species $\phi(\eta)$ with $\eta$ for different values of $\mathrm{m}$

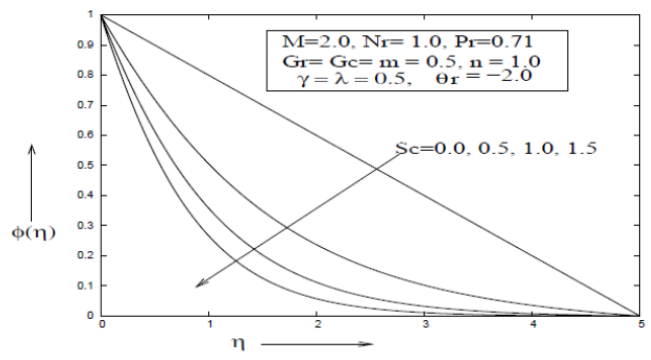

Fig. 19. Influence of concentration species $\phi(\eta)$ with $\eta$ for different values of Sc

The effect of the imposition of various parameters on concentration profiles are shown in Figs. 17 to Fig. 21. It is observed from Fig. 17 that the concentration species $\phi(\eta)$ increases with the increase of the magnetic parameter $M$, whereas the concentration species has a reducing effect on the Hall parameter $m$, 
G.C. Shit and R. Haldar / JAFM, Vol. 5, No. 2, pp. 113-121, 2012.

Table 1 Numerical values of the local skin-friction coefficient $C_{f}=f^{\prime \prime}(0)$

\begin{tabular}{|ccccccc|c|c|c|}
\hline$P r$ & $N r$ & $m$ & $\theta_{r}$ & $\gamma$ & $\lambda$ & $S c$ & $M=0.0$ & $M=2.0$ & $M=4.0$ \\
\hline 0.71 & 1.0 & 0.5 & -2.0 & 0.5 & 0.5 & 0.5 & -0.52597 & -1.45996 & -2.10233 \\
1.0 & & & & & & & -0.55356 & -1.47541 & -2.11350 \\
3.0 & & & & & & & -0.66289 & -1.55043 & -2.17159 \\
\hline 0.71 & 0.5 & 0.5 & -2.0 & 0.5 & 0.5 & 0.5 & -0.49627 & -1.44462 & -2.09153 \\
& 1.0 & & & & & & -0.52596 & -1.45996 & -2.10233 \\
& 5.0 & & & & & & -0.57792 & -1.49009 & -2.12436 \\
\hline 0.71 & 1.0 & 1.0 & -2.0 & 0.5 & 0.5 & 0.5 & -0.52596 & -1.22516 & -1.76392 \\
& & 3.0 & & & & & -0.52596 & -0.73786 & -1.00484 \\
& & 5.0 & & & & & -0.52596 & -0.61575 & -0.75757 \\
\hline 0.71 & 1.0 & 0.5 & -1.0 & 0.5 & 0.5 & 0.5 & -0.56098 & -1.66484 & -2.41425 \\
& & & -2.0 & & & & -0.52596 & -1.45996 & -2.10233 \\
& & & -5.0 & & & & -0.49443 & -1.31365 & -1.88306 \\
\hline 0.71 & 1.0 & 0.5 & -2.0 & 0.1 & 0.5 & 0.5 & -0.49746 & -1.44034 & -2.08909 \\
& & & & 0.5 & & & -0.52596 & -1.45996 & -2.10233 \\
& & & & 1.0 & & & -0.55088 & -1.47691 & -2.11402 \\
\hline 0.71 & 1.0 & 0.5 & -2.0 & 0.5 & -0.5 & 0.5 & -0.42172 & -1.38284 & -2.04079 \\
& & & & & 0.0 & & -0.48498 & -1.43029 & -.07862 \\
& & & & & 0.5 & & -0.52596 & -1.45996 & -2.10233 \\
\hline 0.71 & 1.0 & 0.5 & -2.0 & 0.5 & -0.5 & 0.5 & -0.52596 & -1.45996 & -2.10233 \\
& & & & & & 1.0 & -0.57899 & -1.48921 & -2.12044 \\
& & & & & & 1.5 & -0.61068 & -1.50989 & -2.13432 \\
\hline
\end{tabular}

Table 2 Numerical values of the local number $N u=-\theta(0)$

\begin{tabular}{|ccccccc|c|c|c|}
\hline$P r$ & $N r$ & $m$ & $\theta_{r}$ & $\gamma$ & $\lambda$ & $S c$ & $M=0.0$ & $M=2.0$ & $M=4.0$ \\
\hline 0.71 & 1.0 & 0.5 & -2.0 & 0.5 & 0.5 & 0.5 & 0.51857 & 0.48436 & 0.46751 \\
1.0 & & & & & & & 0.61651 & 0.57475 & 0.55368 \\
3.0 & & & & & & & 1.09706 & 1.03107 & 0.99413 \\
\hline 0.71 & 0.5 & 0.5 & -2.0 & 0.5 & 0.5 & 0.5 & 0.41891 & 0.39371 & 0.38159 \\
& 1.0 & & & & & & 0.51857 & 0.48436 & 0.46751 \\
& 5.0 & & & & & & 0.70871 & 0.66088 & 0.63618 \\
\hline 0.71 & 1.0 & 1.0 & -2.0 & 0.5 & 0.5 & 0.5 & 0.51857 & 0.48973 & 0.47213 \\
& & 3.0 & & & & & 0.51857 & 0.50769 & 0.49279 \\
& & 5.0 & & & & & 0.51857 & 0.51382 & 0.50509 \\
\hline 0.71 & 1.0 & 0.5 & -1.0 & 0.5 & 0.5 & 0.5 & 0.51673 & 0.48081 & 0.46364 \\
& & & -2.0 & & & & 0.51857 & 0.48436 & 0.46751 \\
& & & -5.0 & & & & 0.52016 & 0.48729 & 0.47070 \\
\hline 0.71 & 1.0 & 0.5 & -2.0 & 0.1 & 0.5 & 0.5 & 0.52097 & 0.48641 & 0.46901 \\
& & & & 0.5 & & & 0.51857 & 0.48436 & 0.46751 \\
& & & & 1.0 & & & 0.51662 & 0.48279 & 0.46639 \\
\hline 0.71 & 1.0 & 0.5 & -2.0 & 0.5 & -0.5 & 0.5 & 0.12334 & 0.02936 & -0.02061 \\
& & & & & 0.0 & & 0.35575 & 0.30484 & 0.27964 \\
& & & & & 0.5 & & 0.57857 & 0.48436 & 0.46751 \\
\hline 0.71 & 1.0 & 0.5 & -2.0 & 0.5 & -0.5 & 0.5 & 0.51857 & 0.48436 & 0.46751 \\
& & & & & & 1.0 & 0.51425 & 0.48156 & 0.46573 \\
& & & & & & 1.5 & 0.51223 & 0.48004 & 0.46469 \\
\hline
\end{tabular}

Table3 Numerical values of the local Sherwood number $S h=-\phi^{\prime}(0)$

\begin{tabular}{|ccccccc|c|c|c|}
\hline$P r$ & $N r$ & $m$ & $\theta_{r}$ & $\gamma$ & $\lambda$ & $S c$ & $M=0.0$ & $M=2.0$ & $M=4.0$ \\
\hline 0.71 & 1.0 & 0.5 & -2.0 & 0.5 & 0.5 & 0.5 & 0.67199 & 0.62481 & 0.60072 \\
1.0 & & & & & & & 0.66905 & 0.62322 & 0.59979 \\
3.0 & & & & & & & 0.66032 & 0.61772 & 0.59634 \\
\hline 0.71 & 0.5 & 0.5 & -2.0 & 0.5 & 0.5 & 0.5 & 0.67544 & 0.62659 & 0.60175 \\
& 1.0 & & & & & & 0.67199 & 0.62481 & 0.60072 \\
& 5.0 & & & & & & 0.66670 & 0.62188 & 0.59898 \\
\hline 0.71 & 1.0 & 1.0 & -2.0 & 0.5 & 0.5 & 0.5 & 0.67199 & 0.63256 & 0.60770 \\
& & 3.0 & & & & & 0.67199 & 0.65744 & 0.63733 \\
& & 5.0 & & & & & 0.67199 & 0.66566 & 0.65406 \\
\hline 0.71 & 1.0 & 0.5 & -1.0 & 0.5 & 0.5 & 0.5 & 0.66593 & 0.61956 & 0.59486 \\
& & & -2.0 & & & & 0.67199 & 0.62481 & 0.60072 \\
& & & -5.0 & & & & 0.67411 & 0.62911 & 0.60553 \\
\hline 0.71 & 1.0 & 0.5 & -2.0 & 0.1 & 0.5 & 0.5 & 0.50642 & 0.43681 & 0.40050 \\
& & & & 0.5 & & & 0.67199 & 0.62481 & 0.60072 \\
& & & & 1.0 & & & 0.83627 & 0.80234 & 0.78493 \\
\hline 0.71 & 1.0 & 0.5 & -2.0 & 0.5 & -0.5 & 0.5 & 0.68296 & 0.63338 & 0.60670 \\
& & & & & 0.0 & & 0.67616 & 0.62786 & 0.60279 \\
& & & & & 0.5 & & 0.67199 & 0.62481 & 0.60072 \\
\hline 0.71 & 1.0 & 0.5 & -2.0 & 0.5 & -0.5 & 0.5 & 0.67199 & 0.62481 & 0.60072 \\
& & & & & & 1.0 & 0.96662 & 0.90329 & 0.86889 \\
& & & & & & 1.5 & 1.19569 & 1.12354 & 1.08281 \\
\hline
\end{tabular}


shown in Fig. 18. The reason behind this fact lies in the Eq. (14) and Eq. (15) where the parameters $M$ and $m$ are connected by the relation of the form $\frac{M}{1+m^{2}}$.

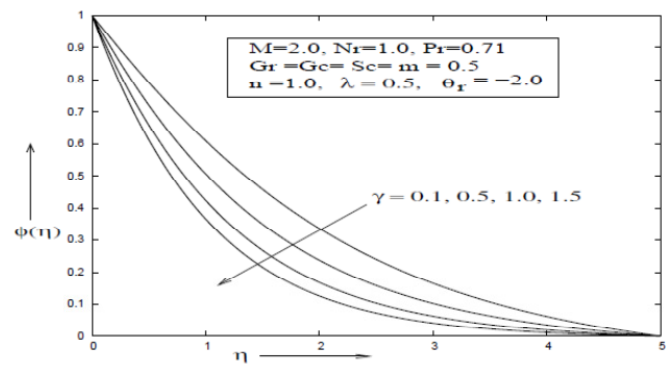

Fig. 20. Influence of concentration species $\phi(\eta)$ with $\eta$ for different values of $\gamma$

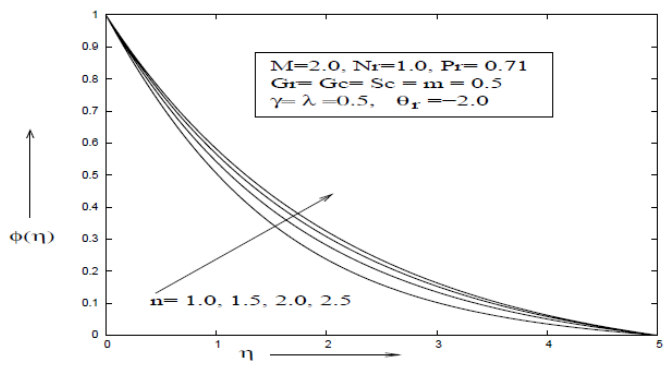

Fig. 21. Influence of concentration species $\phi(\eta)$ with $\eta$ for different values of $n$

As the variation of concentration profiles for different values of the parameters $N r$ and $\theta_{r}$ agrees with the temperature profiles, we have not presented those results. However, Fig. 19 depicts the variation of Schmidt number $S c$ on the species concentration $\phi(\eta)$. It is observed that the species concentration decreases with the increase of the Schmidt number $S c$. Physically this shows that the increase of $S c$ causes decrease of molecular diffusion $D$. The influence of chemical reaction parameter $\gamma$ on the species concentration profiles for generative chemical reaction is shown in Fig. 20. It is noticed that the increasing values of the chemical reaction parameter $\gamma$ is to decelerate the concentration distribution in the boundary-layer. This is due to fact that destructive $(\gamma>0)$ chemical reaction decreases the shortest boundary-layer thickness and thereby increasing the species concentration. Figure 21 indicates that the species concentration increases with the increase of the order of chemical reaction parameter $n$. Therefore, an increase of the chemical reaction parameter enhances the concentration boundary-layer thickness.

The problems of engineering interest for the present study are the local skin-friction coefficient $C_{f}$, the local Nusselt number $N u$ and the local Sherwood number $S h$, which indicates physically the wall shear stress, the rate of heat transfer at the sheet and the rate of mass transfer respectively. The expressions of these physical quantities have been presented in Eq. (20) - Eq. (22). Tables 1-3 exhibit the numerical values to the local skinfriction coefficient $f^{\prime \prime}(0)$, the local Nusselt number $-\theta^{\prime}(0)$ and the local Sherwood number $-\phi^{\prime}(0)$ respectively. It has been observed that for any particular values of the parameters $P r, N r, m, \theta r, \gamma, \lambda$ and $S c$, the local skin-friction coefficient, the local Nusselt number and the local Shearwood number decreases with the increase of the magnetic parameter $M$. Table 1 shows that for any fixed value of $M$, the local skin-friction $C_{f}$ decreases with increasing values of the Prandatl number $\mathrm{Pr}$, thermal radiation parameter $\mathrm{Nr}$, viscosity parameter $\theta r$, the rate of chemical reaction $\gamma$, heat generation/absorption parameter $\lambda$ and the Schmidt number $S c$. Physically, we can say that for the increasing values of the thermal radiation parameter $\mathrm{Nr}$ leads to decreasing of the boundary layer thickness. However, the skin-friction coefficient increases as the Hall parameter $m$ increases. It is worthwhile to mention from Table 2 that the rate of heat transfer at the sheet increases with the increase of the parameters $\mathrm{Pr}, \mathrm{Nr}, \mathrm{m}$ and $\gamma$. The increase of the thermal radiation parameter $\mathrm{Nr}$ has an enhancing effect on the thermal boundarylayer thickness. It is also noticed that the increasing values of the parameters $\theta_{r}, \gamma$ and $S c$ leads to the reduction in heat transfer rate. From these results we observe that the rate of change of heat transfer is insignificant. The rate of mass transfer increases significantly when the Hall parameter $m$, the chemical reaction parameter $\gamma$ and the Schmidt number $S c$ increases. Moreover, the rate of mass transfer decreases insignificantly with the increase of the parameters $\mathrm{Pr}, \mathrm{Nr}$ and $\lambda$.

\section{Conclusion}

In the present investigation, we dealt with the combined effects of thermal radiation and Hall current on the boundary-layer fluid flow, heat and mass transfer with temperature dependent viscosity. From our computed numerical results we observed that the magnetic field and Hall current produce opposite effects on the velocity distribution and heat transfer as well as on the concentration distribution. For a fixed value of $M$, the skin-friction increases with an increase in $m$ and similar is the observation for heat and mass transfer rate. Sufficiently strong heat generation parameter may alter the temperature gradient. The non-dimensional temperature decreases and the concentration increases with increasing values of the Prandtl number Pr. But a reversal trend is observed when the values of the thermal radiation parameter increases. The species concentration decreases with an increase in the values of the Schmidt number $S c$ and chemical reaction parameter 1 whereas opposite trend is observed in the case of the order of the chemical reaction. It is hoped that the results obtained will serve as a scientific tool for understanding more complex flow problems and provide more useful information for engineering applications.

\section{ACKNOWLEDGEMENTS}

The authors are thankful to the DST, New Delhi for the financial support of this investigation through DSTPURSE program of Jadavpur University.

\section{REFERENCES}

Afify, A.A. (2004). MHD free-convective flow and mass transfer over a stretching sheet with chemical reaction. Heat Mass Transfer 40, 495-500.

Andersson, H.I. (1992). MHD flow of a viscoelastic 
fluid past a stretching surface. Acta Mechanica 95, 227-230.

Andersson, H.I., K.H. Bech and B.S. Dandapat (1992). Magneto hydrodynamic flow of a Powerlaw fluid over a stretching surface. International Journal of Non-Linear Mechanics 27, 929-936.

Cebeci, T. and, J. Cousteix (1999). Modelling and computation of boundary-layer flows, SpringerVerlag.

Chakrabarty, A. and A.S. Gupta (1979). Hydromagnetic flow of heat and mass transfer over a stretching sheet. Quarterly of Applied Mathematics 37, 73-78.

Chamakha, A.J. (2003). MHD flow of a uniformly stretched vertical permeable surface in the presence of heat generation/absorption and a chemical reaction. International Communications in Heat and mass transfer 30, 413-422.

Char, M.I. (1994). Heat transfer in a hydromagnetic flow over a stretching sheet. Heat and Mass Transfer 29, 495-500.

Cowling, T.G. (1957). Magneto hydrodynamics. Interscience Publishers, New York.

Crane, L.J. (1970). Flow past a stretching sheet. Journal of Applied Mathematics and Physics (ZAMP) 21, 645-647.

Gupta, P.S. and A.S. Gupta (1977). Heat and Mass Transfer on a stretching sheet with suction or blowing. Canadian Journal of Chemical Engineering 55, 744-746.

Lai, F.C. and F.A. Kulacki (1990). The effect of variable viscosity on convective heat transfer along a vertical surface in a saturated porous mediu. International Journal of Heat and Mass Transfer 33, 1028-1031.

Mahmoud, M.A.A. (2009). Thermal radiation effect on unsteady MHD free convection flow past a vertical plate with temperature-dependent viscosity. Canadian Journal of Chemical Engineering 87, 4752.

Mohamed, R.A. and S.M. Abo-Dahab (2009). Influence of chemical reaction and thermal radiation on the heat and mass transfer in MHD micropolar flow over a vertical moving porous plate in a porous medium with heat generation. International Journal of Thermal Sciences 48, 1800-1813.

Mukhopadhaya, S., G.C. Layek and A. Samad (2005). Study of MHD boundary layer flow over a heated stretching sheet with variable viscosity. International Journal of Heat and Mass Transfer 48, 4460-4466.

Muthucumarswamy, R. and P. Ganesan (2001). Effect of the chemical reaction and injection on flow characteristics in an unsteady upward motion of an isothermal plate. Journal of Applied Mechanics and Technical Physics 42, 665-671.

Pavlov, K.B. (1974). Magnetohydrodynamic flow of an incompressible viscous fluid caused by deformation of plane surface. Magnitnaya Gidrodinamika 4, 146-147.

Raptis, A. and C. Perdikis (1998). Viscoelastic flow by the presence of radiation. Journal of Applied flow by the presence of radiation, Journal of Applied Mathematics and Mechanics 78, 277- 279.

Sakiadis, B.C. (1961). Boundary layer behavior on continuous solid surfaces. American Institute of Chemical Engineering Journal (AIChE) 7, 26-28.

Salem, A.M. (2007). Variable viscosity and thermal conductivity effects on MHD flow and heat transfer in viscoelastic fluid over a stretching sheet. Physics Letters A 369, 315-322.

Sharma, P.R. and G. Singh (2009). Effects of variable thermal conductivity and heat source/sink on MHD flow near a stagnation point on a linearly stretching sheet. Journal of Applied Fluid Mechanics 2(1), 1321.

Shateyi, S. (2008). Thermal radiation and buoyancy effects on heat and mass transfer over a semiinfinite stretching surface with suction and blowing. Journal of Applied Mathematics 2008, Article ID 414830.

Shit, G.C. (2009). Hall effects on MHD free convective flow and mass transfer over a stretching sheet. International Journal of Applied Mathematics and Mechanics 5(8), 22-38.

Watanabe, T. and I. Pop (1995). Hall effects on magnetohydrodynamic boundary layer flow over a continuous moving flat plate. Acta Mechanica 108, $35-47$.

Watanabe, T., and I. Pop (1993). Magnetohydro freeconvection flow over a wedge in the presence of a transverse magnetic field. International Communications in Heat and Mass transfer 20, 871-881. 


\section{Correction:}

Please note that in the hard copy of JAFM Volume 4 Number 2, Special Issue, in the paper entitled: "Modeling and Simulation of Interfacial Turbulent Flows", by Shirani, E., F. Ghadiri and A. Ahmadi, the following sentence is missing and should be added before "Conclusion".

"Note that the DNS results shown in Figs. 7 to 10 are taken from Vincent et al. (2006) and Vincent et al. (2008) and the experimental results shown in Fig. 6 is taken from Sallam et al. (1999)."

\section{References:}

Vincent, S., J. Larocque, D. Lacanette, A. Toutant, P. Lubin, and P. Sagaut (2008). Numerical Simulation of Phase Separation and a Priori Two-Phase LES Filltering. Computers \& Fluids Journal 37, 898-906.

Vincent, S., J. Larocque, D. Lacanette, A. Toutant, P. Lubin, O. Lebaigue, E. Labourasse, J.-P. Caltagirone and P. Sagaut (2006). Governing Equations and a Priori Tests for the LES of Two-Phase Flows. Conference on Turbulence and Interactions, TI2006. 